\begin{abstract}
In this paper, we consider a family of complete or incomplete financial models such that the price processes of the financial assets converge in distribution to those in a limit model. Different authors pointed out that we do not have necessarily convergence of the arbitrage pricing intervals in that context. We prove here that we have very good convergence properties for the equilibrium pricing interval as defined by Bizid, Jouini and Koehl (1998) in discrete time or Jouini and Napp (1999) in continuous time.
\end{abstract}




\title{
Convergence of the equilibrium prices in a family of financial models
}

\author{
Elyès Jouini ${ }^{1}$, CEREMADE, Université Paris IX Dauphine and CREST
}

\section{Introduction}

It is well-known that, in a complete financial market, there is a unique price compatible with the no-arbitrage condition for each asset. That price corresponds to the cost of any replicationg strategy. In that context, if we consider a family of complete markets converging in some sense to another complete market, it suffices to establish good convergence properties on the gain processes in order to prove good convergence properties on the arbitrage prices. Duffie and Protter (1992) present such convergence results on the gain processes developed by Jakubowski, Mémin and Pagès (1989) and Kurtz and Protter (1991, a and b) and they adapt them for easy applications in finance.

Even if their results are valid in the incomplete markets framework they do not give any information about the convergence of the arbitrage interval. Hubalek and Schachermayer (1998) provides some results about the convergence of the arbitrage interval but they rely on the mathematical concept of contiguity, or the financial concept of quasi-arbitrage where, losely speaking, a quasi-arbitrage is, like in the definition of a free-lunch, a sequence of strategies converging to an arbitrage, but where the $n^{t h}$ strategy is applied in the $n^{\text {th }}$ market. It is difficult then to give a "real world" interpretation of these quasi-arbitrages since we can't observe them in anyone of the considered markets. However this concept makes sense if the $(n+1)^{t h}$ market can be embedded in the $n^{t h}$ one. This is the case when the considered sequence of financial markets is introduced in order to modelize large financial markets. This is not the aim of this paper where the sequence of financial markets is introduced as an approximation of the limit market.

In fact, even in very simple and classical situations (stationary trinomial or quadrinomial trees), we can construct sequences of models satisfying all the conditions of Duffie and Protter (1992) and converging in distribution to a Black and Scholes model with a given volatility $\sigma$, but where the arbitrage interval for a given call option does not converge to the Black and Scholes price associated to the volatility $\sigma$ (see Examples 1 and 2).

Nevertheless as underlined by Duffie and Protter "although a large part of financial economic theory is based on models with continuous-time security trading, it is widely felt that these models are relevant insofar as they characterize the behavior of models in

1 The author wants to thank an anonymous referee for careful reading, valuable comments and improvements. Part of this paper was written when the author was visiting the Stern School of Business at New-York University. 
which trade occur discretely in time". It seems then natural to look for pricing intervals with good convergence properties.

In this paper, we prove that the equilibrium pricing interval introduced by Bizid, Jouini and Koehl (1999) in a discrete time framework and by Jouini and Napp (1999) in a continuous time framework has these good convergence properties.

This interval is defined as the set of all the expected values of the terminal payoff of a given asset with respect to all the equivalent probability measures with densities "in reverse order" than the total wealth of the economy.

Our restrictions on the family of the probabilities used to price the derivative asset with respect to the classical case are similar to those introduced by Perrakis and Ryan (1984) and Perrakis $(1986,1993)$. In those papers, the authors propose an ordering principle on the probabilities used to price the derivative asset. In Bizid, Jouini and Koehl (1999) and Jouini and Napp (1999) this ordering principle concerns the densities instead of the probabilities and is derived from the equilibrium theory, and more precisely from the market clearing conditions. If we assume that all the states of the world are equiprobable, our result permits then to establish the convergence for the Perrakis (1986) prices.

This result is a generalization of Perrakis (1993) where the author proves the convergence of the "well ordered" prices in a multinomial model where the distribution of the successive returns stay the same for every period and where the price process converges in distribution to the Black and Scholes (1973) one or to the Merton (1976) mixed diffusion-jump one.

The paper is organized as follows. In section 2, we introduce the main concepts and result and in section 3 we prove a Ranga Rao (1962) type Lemma for sequences of nonincreasing functions instead of equicontinuous ones and we prove our main result as a consequence of this key Lemma.

\section{Equilibrium pricing interval}

Before introducing our general framework, let us consider the simplest example of incomplete markets and let us explain the main idea of the so-called equilibrium interval. More precisely, consider a simple one-period model where the sample space and the probability are $\Omega=\left\{\omega_{1}, \omega_{2}, \omega_{3}\right\}$ and $P=(1 / 3,1 / 3,1 / 3)$.

First, there are two assets in the market: a first asset whose prices are $p(0)=25$, at date 0 and $p\left(\omega_{1}\right)=20, p\left(\omega_{2}\right)=30$ or $p\left(\omega_{3}\right)=40$ at date 1 and a risk-free asset defined by an interest rate $r=\frac{1}{19}$.

It is straightforward that the feasible range of the density of the martingale measures with respect to $P$ is $\left(Q\left(\omega_{1}\right), Q\left(\omega_{2}\right), Q\left(\omega_{3}\right)\right)=\left(0.368+Q\left(\omega_{3}\right), 0.632-2 Q\left(\omega_{3}\right), Q\left(\omega_{3}\right)\right)$ where $Q\left(\omega_{3}\right) \in[0,0.316]$.

If there is a representative agent in this economy, the maximization program of this agents leads to the following first-order conditions:

$$
\left(Q\left(\omega_{1}\right), Q\left(\omega_{2}\right), Q\left(\omega_{3}\right)\right)=\left(\frac{u^{\prime}(20)}{u^{\prime}(25)}, \frac{u^{\prime}(30)}{u^{\prime}(25)}, \frac{u^{\prime}(40)}{u^{\prime}(25)}\right)
$$


and since $u$ is usually strictly-concave, we must have

$$
Q\left(\omega_{1}\right)>Q\left(\omega_{2}\right)>Q\left(\omega_{3}\right) .
$$

After imposing these restrictions, we get the feasible range of the risk-neutral probability $Q\left(\omega_{3}\right) \in[0.088,0.211]$. Consequently this approach permits to obtain bounds on the price of the derivative assets, which are better than the usual arbitrage-free bounds.

Following the same idea, the option bounding approach-introduced by Perrakis and Ryan (1984) and extended by Perrakis $(1986,1993)$ - derives upper and lower limits on option prices by assuming that stock returns and risk-neutral probability are in reverse order.

Bizid, Jouini and Koehl (1999) and Jouini and Napp (1999) proposed to replace the risk neutral probability by the density of such a probability with respect to the historical one and called "equilibrium bounds" the bounds obtained by assuming that stock returns and risk-neutral density are in reverse order. Furthermore they proved, in different settings, that this ordering principle is a necessary condition for the derivatives prices to be compatible with an equilibrium.

From an empirical point of view, Aït-Sahalia and Lo $(1997,1998)$ propose a nonparametric estimation of the state-price deflators. Using the market information, they estimate an option-pricing formula, then differentiate twice this estimator with respect to the strike of the option. Under suitable regularity conditions, this last quantity converges to a state-price deflator. Their empirical results confirm our theoretical "ordering principle".

Let us now define carefully the equilibrium interval:

Definition 1 A financial model $M$ is defined by:

- a finite horizon date $T$,

- a probability space $(\nless, \mathcal{F}, P)$,

- a filtration $\left(\mathcal{F}_{t}\right)_{t \in[0, T]}$ on $(\nless, \mathcal{F}, P)$ satisfying the usual assumptions of right continuity and completeness,

- a set $S$ of assets with positive càdlàg, adapted, price processes denoted by $\left\{s^{i}\right\}_{i=0, \ldots, m}$;

In all this section, we will assume that one of the assets (namely $s^{0}$ ) is risk-free and that it has a constant price equal to 1 . This last assumption is made only in order to normalize the price processes and has no impact on our results. Let $p$ be in $\{0, \cdots, m-1\}$ and let us assume that assets $0, \cdots, p$ are in zero net supply and that assets $p+1, \cdots, m$ are in positive supply and for each one of them we will normalize its supply to one.

For a given vector $x$ we define $W(x)$ as the sum of the last $(m-p)$ coordinates.

For a given date $t$ the total wealth at $t$ is then defined by

$$
W_{t}=W\left(\left(s^{i}(t)\right)_{i=0, \cdots, m}\right)=\sum_{i=p+1}^{m} s^{i}(t)
$$

and the sum is then extended over all the assets with positive (and then normalized to one) net supply. 
Before to introduce our main definitions and results let us introduce some mathematical tools.

We denote by $\mathbb{D}^{m}$ the space of $\mathbb{R}^{m}$ - valued càdlàg sample paths on a fixed interval $\mathcal{T}=[0, T]$ endowed with the Skorokhod topology and by $\mathcal{D}^{m}$ the $\sigma$-field generated by the open sets of $\mathbb{D}^{m}$. We refer to Jacod and Shiryaev, (1987, chapter VI), for the definitions of these concepts as well as for the definition of the convergence in distribution of a sequence of $\mathbb{R}^{m}$-valued càdlàg processes $X_{n}$ to another process $X_{\infty}$, denoted by $X_{n} \Longrightarrow X_{\infty}$.

For a given càdlàg adapted stochastic process $X$ and a given stopping time $\tau$ we will denote by $X^{\tau}$ the process $X$ defined by

$$
X^{\tau}(t, \omega)=\left\{\begin{array}{c}
X(t, \omega) \text { if } t<\tau(\omega) \\
\lim _{t^{\prime} \rightarrow \tau(\omega), t^{\prime}<\tau(\omega)} X\left(t^{\prime}, \omega\right) \text { if } t \geq \tau(\omega)
\end{array}\right.
$$

and, for a given positive number $b$ by $X^{b}$ the stopped process $X^{\tau^{b}}$ where $\tau^{b}$ is defined by

$$
\tau^{b}(\omega)=\inf \{t \geq 0,\|X(t, \omega)\| \geq b\}
$$

and where the infimum is supposed to be equal to $T$ when the associated set is empty.

Since we assumed that $X$ is càdlàg, we have then that $W\left(X^{b}\right)$ is bounded above for $b$ sufficiently large. In the next $W\left(X^{b}\right)$ will be denoted by $W^{b}$ and $W\left(X^{\tau}\right)$ by $W^{\tau}$.

Definition 2 Let us consider a given positive number $B$. We define the set $\Gamma^{B}$ as the set of equivalent martingale-measures $Q$ for $\left\{s^{i}\right\}_{i=0, \ldots, m}$ such that, for all stopping time $\tau$, there exists a nonincreasing, positive function $g_{\tau} \leq B$ such that

$$
E_{\tau}\left[\frac{d Q}{d P}\right]=g_{\tau}\left(W^{\tau}\right) .
$$

In the next $B$ is kept fixed and we denote by $\Gamma$ the set $\Gamma^{B}$, by $\Gamma^{l o c}$ the set of probability measures satisfying the same conditions as those in $\Gamma$ except that the priceprocesses are local-martingales instead of martingales. We will also denote by $\Gamma^{l o c, a c}$ the set of probability measures satisfying the same conditions as those in $\Gamma^{l o c}$ except that the functions $\left(g_{\tau}\right)_{\tau}$ are only nonnegative instead of positive (in particular, $Q$ is then absolutely continuous with respect to $P$ instead of equivalent).

If we introduce now a new purely-financial asset in the market $M$, which final payoff $q_{T}$ is known, the equilibrium interval is defined as follows:

$$
E I(q)=\left\{E^{Q}\left[q_{T}\right]: Q \in \Gamma\right\} .
$$

Note that, if this "new" asset is already in $S$ then, the previous interval is reduced to one point : the known initial price of that asset.

Assume now that we have a sequence of financial models $\left(M_{n}\right)_{n=0, \cdots, \infty}$ on a sequence of probability spaces $\left(\Omega_{n}, F_{n}, P_{n}\right)_{n=0, \cdots, \infty}$ and let us assume that they have the same number $m$ of assets, the same number $p+1$ of zero-net supply assets, the same number $m-p$ of normalized to one net supply assets, the same horizon $T$ and let us denote by $X_{n}$ the $\mathbb{R}^{m}$-valued process defined by $X_{n}=\left(s_{n}^{i}\right)_{i=1, \cdots, m}$. We will also 
assume that $X_{n}(0)$ does not depend on $n$ and we will denote it by $X(0)$ (for instance, we can assume that the initial prices of all the initially marketed assets and in all the models are normalized to one).

We are now ready to define the convergence of models as follows:

Definition 3 We say that the sequence $\left(M_{n}\right)_{n \in \mathbb{N}}$ converges to a model $M_{\infty}$ (and we denote it by $\left.M_{n} \Rightarrow M_{\infty}\right)$ if $X_{\infty}$ is locally bounded, if the filtration of $M_{\infty}$ is generated by $X_{\infty}$ and if

$$
X_{n} \Rightarrow X_{\infty}
$$

Proposition 1 (Example) If $M_{n}$ is the Cox-Ross-Rubinstein (1979) binomial model with one stock and $n$ dates on a given time interval $[0, T]$ and if the mean and variance of the logarithmic return of the stock by unit of time converge respectively to $\mu$ and $\sigma$ then the sequence $\left(M_{n}\right)$ converges to the Black and Scholes (1973) model with parameters $\mu$ and $\sigma$.

In order to prove the convergence in distribution in that case we refer to Duffie (1988, section 22).

Remark that we can not apply here the results of Duffie and Protter (1992) about the convergence of financial gain processes since we consider arbitrage prices or equilibrium prices and not replicating prices. Then our prices are not directly linked to any gain process for any strategy.

It is well known that in the Cox-Ross-Rubinstein example, the price of any derivative asset (defined by a final payoff equal to a given function of the final payoff of the stock) converges to the price of the derivative asset defined by the same function in the limit-model (see e.g. Duffie and Protter 1992).

It is well known too that even if the models $\left(M_{n}\right)$ are incomplete as in a trinomial model with only one stock, the limit model can in certain cases be complete. Nevertheless, Prigent (1994) showed on some examples that the arbitrage price interval of a given derivative asset in $\left(M_{n}\right)$ does not necessarily converge to the unique price in the limit complete market model. In fact, the arbitrage price interval converges, in general, to a given interval containing the unique price in the limit model.

Let us present here one of his examples.

Example 2 (Prigent) We consider a sequence of stationary trinomial models where the returns at each node are $1+\frac{\tau}{n}+\frac{\alpha_{i}}{n^{1 / 2}}$ with positive probabilities $p_{i}$ for $i=1,2,3$. We further assume that $\tau>0, \alpha_{1}>\alpha_{2}>0,1+\tau>-\alpha_{3}>\tau$

- If $\sum_{i=1}^{3} p_{i} \alpha_{i}=0$, this model converges to the Black-Scholes model with volatility $\sqrt{\sum_{i=1}^{3} p_{i} \alpha_{i}^{2}}$.

- The set of martingale measures in $M_{n}$ has at each node exactly two extremal transition martingale-measures on the form $\underline{\pi}=\left(0, \underline{\pi}_{2}, \underline{\pi}_{3}\right)$ and $\bar{\pi}=\left(\bar{\pi}_{1}, 0, \bar{\pi}_{3}\right)$. 
- The extremal price of a call option ${ }^{2}$ is obtained using always the same extremal transition probability at each node.

- For a call option the arbitrage interval converges to $\left[B S\left(\sqrt{-\alpha_{2} \alpha_{3}}\right), B S\left(\sqrt{-\alpha_{1} \alpha_{3}}\right)\right]$ (where $B S$ is the Black-Scholes formula considered as a function of the volatility).

The two next examples are from Bizid, Jouini and Koehl (1998). The first one is a discretization of a continuous time model and we study the convergence when the time step goes to zero. The second one is parametrized by a continuous parameter and we study the convergence of the equilibrium interval when the parameter goes to infinity for a given fixed size of the tree.

Example 3 (Bizid-Jouini-Koehl) We consider a sequence of stationary quadrinomial models with equiprobable returns at each node $e^{r \frac{T}{n}+\sigma_{i}} \sqrt{\frac{T}{n}}, i=1, \cdots, 4$ and where we have $\sigma_{1}=-\sigma_{4} \stackrel{\text { def }}{=} \sigma_{\max }>\sigma_{2}=-\sigma_{3} \stackrel{\text { def }}{=} \sigma_{\text {min }}$.

- The arbitrage interval for a call option converges to $\left[B S\left(\sigma_{\min }\right), B S\left(\sigma_{\max }\right)\right]$ as in Avellaneda, Levy and Paras (1995).

- The model converges, when n goes to infinity, to the Black and Scholes model with volatility $\sqrt{\frac{\sigma_{\min }^{2}+\sigma_{\max }^{2}}{2}}$.

- With $\sigma_{\min }=10 \%, \sigma_{\max }=15 \%, T=1$ year, $r=5 \%, n=500$ and $^{3} S_{0}=K=$ 100 , the arbitrage interval is $[6.812824 ; 8.602021]$, the equilibrium interval is [7.770313;7.779874] for $K$ large enough and the price in the limit model is 7.764116 .

In the next, a derivative asset in the model $M_{n}$ will be defined as an asset with a payoff equal to a given real-valued continuous function of $X_{n}=\left(X_{n}(t)\right)_{0<t<T}$. Our derivative asset has then a payoff described by a function of the paths of the primitive assets processes in the model.

In the next we will denote by $q_{n}(T)=F_{n}\left(X_{n}\right)$, the final payoff of a given derivative asset. The equilibrium interval for this asset is the defined by

$$
E I_{n}\left(F_{n}\right)=\left\{E^{Q}\left[F_{n}\left(X_{n}\right)\right]: Q \in \Gamma_{n}\right\} .
$$

The interval $E I_{n}^{l o c}\left(F_{n}\right)\left(\right.$ resp. $\left.E I_{n}^{l o c, a c}\left(F_{n}\right)\right)$ will be defined exactly as $E I_{n}\left(F_{n}\right)$ replacing $\Gamma_{n}$ by $\Gamma_{n}^{l o c}$ (resp. $\left.\Gamma_{n}^{l o c, a c}\right)$.

Furthermore, we will say that a sequence of intervals $I_{n}$ converges in a given interval $I_{\infty}$ and we will denote it by $\lim _{n} I_{n} \hookrightarrow I_{\infty}$ in order to say that, for all convergent sequence $\left(u_{n}\right)$ in $I_{n}$, we have $\lim u_{n} \in I_{\infty}$.

We can now apply these definitions and results in order to show a convergence result on derivatives prices :

2 Or any other derivative asset defined by a convex function of the terminal payoff of the primitive asset

3 Where, as usual, $K$ is the option strike and $S_{0}$ the initial price of the underlying asset. 
Theorem 4 Assume that $M_{n} \Rightarrow M_{\infty}$ and let us introduce a derivative asset defined by $q_{n}(T)=F_{n}\left(X_{n}\right)$ in the model $M_{n}$ (for $\left.n=0, \cdots, \infty\right)$ where $F_{n}$ is a sequence of bounded and continuous (for the Skorokhod topology) functions converging uniformly to a given function $F_{\infty}$, then (i) we have:

$$
\lim _{n} E I_{n}\left(F_{n}\right) \hookrightarrow E I_{\infty}^{l o c, a c}\left(F_{\infty}\right)
$$

(ii) if $E I_{\infty} \neq \emptyset$ then

$$
\lim _{n} E I_{n}\left(F_{n}\right) \hookrightarrow \overline{E I_{\infty}^{l o c}\left(F_{\infty}\right)}
$$

(iii) if $E I_{\infty} \neq \emptyset$ and $\left(X_{\infty}\right)$ is of class ${ }^{4}(D)$, then

$$
\lim _{n} E I_{n}\left(F_{n}\right) \hookrightarrow \overline{E I_{\infty}\left(F_{\infty}\right)}
$$

(iv) finally, if $E I_{n} \neq \emptyset$ for $n$ sufficiently large and if $M_{\infty}$ is complete and arbitragefree (more accurately, such that there exists one and only one equivalent martingalemeasure), then

$$
\lim _{n} E I_{n}\left(F_{n}\right)=E I_{\infty}\left(F_{\infty}\right)
$$

Remark 1 Under the conditions of (iii) or (iv) and if $F$ is not bounded but such that $F(x)=\alpha x \cdot e$ for a given real number $\alpha$, a given vector $e$ in $\mathbb{R}^{m}$ and for $x$ sufficiently large, then it suffices to apply our result to $q-\alpha \sum_{i=1}^{m} e_{i} s^{i}$ (Put-Call Parity).

\section{Proof of the Theorem}

In order to prove the theorem we will follow the following steps :

- By stopping the problem is reduced to a process which is bounded from above.

- It is shown that the martingale measures sequence $\left(Q_{n}\right)$ is tight and thus there is a limit candidate measure.

- Boundedness and monotonicity yields a Helly-type selection theorem...

- ... that permits to prove a Ranga Rao (1962)-type Theorem (see also Billingsley, $1968, \mathrm{p} 17$ ) for a family of decreasing functions (instead of equicontinuous ones). This is in fact the key result of the paper.

- The limit measure is then a martingale-measure.

- By stopping and approximation arguments the result extends to the original sequence of processes.

4 i.e. the family $\left(X_{\infty}^{\tau}\right)_{\tau \in \Upsilon}$, where $\Upsilon$ is the set of all finite value stopping times, is uniformly integrable, see Jacod and Shiryaev, 1987, p11. 


\subsection{Reduction to bounded processes}

Since $X_{\infty}$ is locally bounded there exists a sequence $b_{k}$ converging to $\infty$ such that $\tau^{b_{k}}$ converges to $T$ and $X_{\infty}^{b_{k}}$ converges to $X_{\infty}$ in probability. Furthermore, $\left(b_{k}\right)$ can be chosen such that $X_{n}^{b_{k}} \Rightarrow X_{\infty}^{b_{k}}$ for all $k$. Indeed, when the set of paths with a jump or a "plateau" when they reach $b$ have a zero measure with respect to $P_{\infty}$ then we have $X_{n}^{b} \Rightarrow X_{\infty}^{b}$. Furthermore, we refer to Jacod and Shiryaev (1987, chapter IX) in order to see that the set of real numbers $b$ for which the set of paths with a jump or a "plateau" when they reach $b$ does not have a zero measure with respect to $P_{\infty}$ is at most countable.

In the next we will omit the subscript $k$ and we consider a sequence of martingalemeasures $Q_{n}$ such that, for all $n, Q_{n}$ belongs to $\Gamma_{n}$. Therefore, there exists a nonincreasing function $g_{n}^{b}$ such that $E_{\tau^{b}}^{P_{n}}\left[\frac{d Q_{n}}{d P_{n}}\right]=g_{n}^{b}\left(W_{n}\left(\tau^{b}\right)\right)$ where $W_{n}$ stands for $W\left(X_{n}\right)$. We will denote by $\mu_{n}^{b}$ the distribution probability of $X_{n}^{b}$.

In the next, $b$ will be, unless specified, kept fixed and we will denote by $\tau$ the stopping time $\tau^{b}$, by $X_{n}$ the process $X_{n}^{b}$, by $g_{n}$ the function $g_{n}^{b}$ and by $\mu_{n}$ the distribution probability $\mu_{n}$. The density of the restriction of $Q_{n}$ to $\left(\Omega, \mathcal{F}_{\tau}\right)$ with respect to $P_{n}$ is then given by $g_{n}$.

\subsection{A tightness result}

By construction, we have

$$
\left\|g_{n}\right\|_{\infty} \leq B
$$

Let us denote by $\tilde{Q}_{n}$ the probability measure with density $g_{n}(W(x(T)))$ with respect to $\mu_{n}$. Let us prove that $\left(\tilde{Q}_{n}\right)$ is tight. By definition (see Billingsley (1968)), it suffices to prove that for all $\varepsilon>0$, there exists a compact set $K$ in $\mathbb{D}^{m}$ (endowed with the Skorokhod topology) such that $\tilde{Q}_{n}(K)>1-\varepsilon$ for all $n$.

But we know that the family $\left(\mu_{n}\right)$ converges weakly and then is tight (Billingsley, 1968, Theorem 29.3). There exists then $K$ such that $\mu_{n}(K)>1-\varepsilon^{\prime}$ for all $n$ with $\varepsilon^{\prime}<\frac{\varepsilon}{B}$. We have then,

$$
\begin{aligned}
\tilde{Q}_{n}\left(K^{c}\right) & =\int_{K^{c}} g_{n}(W(x(T))) d \mu_{n}(x) \\
& \leq B \int_{K^{c}} d \mu_{n}(x) \leq B \varepsilon^{\prime}<\varepsilon
\end{aligned}
$$

The family $\left(\tilde{Q}_{n}\right)$ is then tight and we can extract from it a subsequence $\left(\tilde{Q}_{\varphi(n)}\right)$ converging weakly to some measure $\tilde{Q}_{\infty}$. In the next we will denote by $\left(\tilde{Q}_{n}\right)$ that subsequence.

We want now to prove that $\tilde{Q}_{\infty}$ has a density with respect to $\mu_{\infty}$ defined by a nonincreasing function of the wealth. For that we need first a Helly-type selection Theorem.

\subsection{A Helly-type selection theorem}


Lemma 5 If $\left(h_{n}\right)$ is a sequence of uniformly bounded real-valued nonincreasing nonnegative functions defined on $[d, b]$ then we can extract a subsequence also denoted by $\left(h_{n}\right)$ converging pointwise on $[d, b]$ to a given real-valued nonnegative nonincreasing function $h_{\infty}$ such that for all u except on a countable set we have

$$
\forall \varepsilon, \exists n_{\varepsilon}^{u}, \exists \eta_{\varepsilon}^{u}: \forall n \geq n_{\varepsilon}^{u},|v-u|<\eta_{\varepsilon}^{u} \Longrightarrow\left|h_{n}(v)-h_{n}(u)\right|<\varepsilon
$$

Helly's (1912) theorem permits us to construct a subsequence $\left(h_{\theta(n)}\right)$ converging on $[d, b]$ to some nonincreasing function $h_{\infty}$.

Now, for all continuity point $u$ of $h_{\infty}$ we have

$$
h_{\theta(n)}(u) \rightarrow h_{\infty}(u) .
$$

and we can find $\eta_{\varepsilon}^{u}$ such that,

$$
\forall v \in[d, b],|u-v|<\eta_{\varepsilon}^{u} \Rightarrow\left|h_{\infty}(u)-h_{\infty}(v)\right|<\varepsilon
$$

furthermore we can chose $\eta_{\varepsilon}^{u}$ such that $u-\eta_{\varepsilon}^{u}$ and $u+\eta_{\varepsilon}^{u}$ are both continuity points for $h_{\infty}$.

Then for $n$ sufficiently large we have

$$
\left|h_{\theta(n)}\left(u+\delta \eta_{\varepsilon}^{u}\right)-h_{\infty}\left(u+\delta \eta_{\varepsilon}^{u}\right)\right|<\varepsilon, \delta=-1,0,1 .
$$

and since all the functions $\left(h_{n}\right)$ are nonincreasing we have, for all $v$ such that $u \leq v \leq$ $u+\eta_{\varepsilon}^{u}$,

$$
\begin{aligned}
\left|h_{\theta(n)}(u)-h_{\theta(n)}(v)\right| & \leq h_{\theta(n)}(u)-h_{\theta(n)}\left(u+\eta_{\varepsilon}^{u}\right) \\
& \leq\left(h_{\theta(n)}(u)-h_{\infty}(u)\right) \\
& +\left(h_{\infty}(u)-h_{\infty}\left(u+\eta_{\varepsilon}^{u}\right)\right) \\
& +\left(h_{\infty}\left(u+\eta_{\varepsilon}^{u}\right)-h_{\theta(n)}\left(u+\eta_{\varepsilon}^{u}\right)\right) \\
& <3 \varepsilon
\end{aligned}
$$

and we have exactly the same result for $v$ such that $u-\eta_{\varepsilon}^{u} \leq v \leq u$.

We have then proved that, there exists $\eta_{\varepsilon}^{u}$ such that, for $n$ sufficiently large and for all $v$ such that $|u-v|<\eta_{\varepsilon}^{u}$ we have

$$
\left|h_{\theta(n)}(u)-h_{\theta(n)}(v)\right|<3 \varepsilon
$$

\subsection{A Ranga Rao-type Theorem}

Lemma 6 If $M_{n} \Rightarrow M_{\infty}$ and if $\left(h_{n}\right)_{n \in \mathbb{N}}$ is a sequence of uniformly bounded realvalued nonincreasing nonnegative functions defined on $[d, b]$, then for all sequence of bounded real-valued, continuous (for the Skorokhod topology) functions $f_{n}$ on $\mathbb{D}^{m}$ 
converging uniformly to a function $f_{\infty}$, there exists a subsequence also denoted $\left(h_{n}\right)$ and a real-valued nonnegative nonincreasing function $h_{\infty}$ defined on $[d, b]$ such that :

$$
\int f_{n}(x) h_{n}(W(x(T))) d \mu_{n} \rightarrow \int f_{\infty}(x) h_{\infty}(W(x(T))) d \mu_{\infty} .
$$

This is in fact the key Lemma of this paper. In order to prove this result we first reduce the problem by considering that $f$ does not depend on $n$. Then, thanks to previous Lemma we provide an equicontinuous-like decomposition of $\mathbb{R}^{+}$. The following step follows the same lines as the original Ranga Rao Theorem (1962) proof and leads to an approximation of our measures by coutable support measures. The last step concludes.

Let $\varepsilon$ be a given positive real number and let us continue to use the same notations as in the previous proof.

\section{- Step 1 : Reduction of the problem}

First remark that

$$
\begin{aligned}
& \left|\int f_{n}(x) h_{n}(W(x(T))) d \mu_{n}-\int f_{\infty}(x) h_{\infty}(W(x(T))) d \mu_{\infty}\right| \\
& \leq\left|\int f_{n}(x) h_{n}(W(x(T))) d \mu_{n}-\int f_{\infty}(x) h_{n}(W(x(T))) d \mu_{n}\right| \\
& +\left|\int f_{\infty}(x) h_{n}(W(x(T))) d \mu_{n}-\int f_{\infty}(x) h_{\infty}(W(x(T))) d \mu_{\infty}\right|
\end{aligned}
$$

and that $h_{n}$ is uniformly bounded, the measures $\mu_{n}$ are probability measures and $\left(f_{n}\right)$ converges uniformly to $f_{\infty}$. The first term on the right of the inequality symbol is then arbitrarily small for $n$ sufficiently large. We will work then on the second term and we will simply denote by $f$ the function $f_{\infty}$.

\section{- Step 2 : Equicontinuous like decomposition}

For all continuity points $u$ for $h_{\infty}$, let us denote by $N_{u}$ the set defined by

$$
N_{u}=\left\{v \in \mathbb{R}:|u-v|<\eta_{\varepsilon}^{u}\right\}
$$

we have then that

$$
\forall v \in N_{u}, \forall n \geq n_{\varepsilon}^{u},\left|h_{n}(u)-h_{n}(v)\right|<\varepsilon
$$

and without any loss of generality, we can assume that $\pi_{T}^{-1} W^{-1}\left(N_{u}\right)$ is a continuity set for $\mu_{\infty}$.

Let us consider $N=\cup N_{u}$ where the union is extended over all the continuity points for $h_{\infty}$. The set $N$ is open and has a countable complement in $\mathbb{R}_{*}^{+\stackrel{\text { def }}{=}} \mathbb{R}^{+} \backslash\{0\}$. It has then a countable number of connected components. Let us consider $\left(w, w^{\prime}\right)$ one of these components. The set $\left[w+\frac{1}{n}, w^{\prime}-\frac{1}{n}\right]$ is compact and can be covered by a finite union of sets $N_{u}$. There exists then a sequence $\left(u_{j}\right)$ such that

$$
\mathbb{R}_{*}^{+}=\bigcup_{j=0}^{\infty} N_{u_{j}} \cup D
$$


where $D$ is a subset of the set of discontinuity points for $h_{\infty}$ and is then finite or countable. We will denote by $\left(a_{k}\right)_{k \in \mathbb{N}}$ the elements of $D$.

If we denote by $C_{0}$ the set $N_{u_{0}}, C_{1}$ the set $N_{u_{1}} \cap C_{0}^{c}$, and so on, we will obtain a countable partition of $\mathbb{R}_{*}^{+} \backslash D$. We have then

$$
\sum_{j=0}^{\infty} \mu_{\infty} \pi_{T}^{-1} W^{-1}\left(C_{j}\right)+\sum_{k=0}^{\infty} \mu_{\infty} \pi_{T}^{-1} W^{-1}\left(\left\{a_{k}\right\}\right)=1
$$

and it exists then $N^{\varepsilon}$ sufficiently large such that

$$
\sum_{j \geq N^{\varepsilon}}^{\infty} \mu_{\infty} \pi_{T}^{-1} W^{-1}\left(C_{j}\right)+\sum_{k \geq N^{\varepsilon}}^{\infty} \mu_{\infty} \pi_{T}^{-1} W^{-1}\left(\left\{a_{k}\right\}\right) \leq \varepsilon .
$$

Furthermore for all $k$, there exists $N_{k}$ sufficiently large such that $\left|h_{n}\left(a_{k}\right)-h_{\infty}\left(a_{k}\right)\right|<$ $\varepsilon$, for all $n \geq N_{k}$. In the next, we will denote by $n_{\varepsilon}$ the integer defined by

$$
n_{\varepsilon}=\sup \left\{\sup _{k \leq N^{\varepsilon}} N_{k}, \sup _{j \leq N^{\varepsilon}} n_{\varepsilon}^{u_{j}}\right\} .
$$

We have then for all $j, k \leq N^{\varepsilon}$, all $n \geq n_{\varepsilon}$, all pair $(u, v)$ in $C_{j}$,

$$
\left|h_{n}(u)-h_{n}(v)\right|<\varepsilon, \quad\left|h_{n}\left(a_{k}\right)-h_{\infty}\left(a_{k}\right)\right|<\varepsilon .
$$

\section{- Step 3 : Approximation by countable support measures}

Let us now consider a family $\left(x_{j}\right)$ in $\mathbb{D}^{m}$ and a family $\left(y_{k}\right)$ in $\mathbb{D}^{m}$ such that for all $j \geq 1$ and all $k \geq 0$, we have $W\left(\pi_{T}\left(x_{j}\right)\right) \in C_{j}$ and $W\left(\pi_{T}\left(y_{k}\right)\right)=a_{k}$, and let us construct, for $n=1, \cdots, \infty$ a measure $\lambda_{n}$ defined on its support $\left\{x_{j}: j \geq 0\right\} \cup$ $\left\{y_{k}: k \geq 0\right\}$ by $\lambda_{n}\left(x_{j}\right)=\tilde{\mu}_{n}\left(C_{j}\right)$, and $\lambda_{n}\left(y_{k}\right)=\tilde{\mu}_{n}\left(a_{k}\right)$ where $\tilde{\mu}_{n}=\mu_{n} \pi_{T}^{-1} W^{-1}$.

If we omit the $x$ 's and if we denote by $W_{T}$ the quantity $W(x(T))$, we have for $n \geq n_{\varepsilon}$ and for $n=\infty$,

$$
\begin{aligned}
& \left|\int f h_{n}\left(W_{T}\right) d \lambda_{n}-\int f h_{n}\left(W_{T}\right) d \mu_{n}\right| \\
& =\sum_{j=0}^{N_{\varepsilon}}\left|\int_{\tilde{C}_{j}} f h_{n}\left(W_{T}\right) d \lambda_{n}-\int_{\tilde{C}_{j}} f h_{n}\left(W_{T}\right) d \mu_{n}\right| \\
& +\sum_{j=N_{\varepsilon}+1}^{\infty}\left|\int_{\tilde{C}_{j}} f h_{n}\left(W_{T}\right) d \lambda_{n}-\int_{\tilde{C}_{j}} f h_{n}\left(W_{T}\right) d \mu_{n}\right| \\
& +\sum_{k=0}^{\infty}\left|f\left(y_{k}\right) h_{n}\left(a_{k}\right) \lambda_{n}\left(\left\{y_{k}\right\}\right)-f\left(y_{k}\right) h_{n}\left(a_{k}\right) \tilde{\mu}_{n}\left(\left\{a_{k}\right\}\right)\right|
\end{aligned}
$$

The last term is, by definition, equal to zero and using the monotonicity of $\left(h_{n}\right)$ we 
have,

$$
\begin{aligned}
\left|\int f h_{n}\left(W_{T}\right) d \lambda_{n}-\int f h\left(W_{T}\right) d \mu_{n}\right| & \leq M \sum_{j=0}^{N_{\varepsilon}} \int_{C_{j}}\left|h_{n}\left(u_{j}\right)-h_{n}(u)\right| d \tilde{\mu}_{n}(u) \\
& +2 M B \sum_{j=N^{\varepsilon}+1}^{\infty} \tilde{\mu}_{n}\left(C_{j}\right) \\
& \leq \varepsilon M \tilde{\mu}_{n}\left(\bigcup_{j=1}^{\infty} C_{j}\right)+2 \varepsilon M B \leq \varepsilon M(1+2 B)
\end{aligned}
$$

where $M$ is a bound on $f$ and $B$ is the uniform bound on the family $\left(h_{n}\right)$.

Furthermore, it is easy to see that for $n \geq n_{0}$

$$
\begin{aligned}
& \left|\int f h_{n}\left(W_{T}\right) d \lambda_{n}-\int f h_{n}\left(W_{T}\right) d \lambda_{\infty}\right| \\
& \leq M B \sum_{j=0}^{\infty}\left|\tilde{\mu}_{n}\left(C_{j}\right)-\tilde{\mu}_{\infty}\left(C_{j}\right)\right|+M B \sum_{k=0}^{\infty}\left|\tilde{\mu}_{n}\left(\left\{a_{k}\right\}\right)-\tilde{\mu}_{\infty}\left(\left\{a_{k}\right\}\right)\right|
\end{aligned}
$$

We have $\tilde{\mu}_{n}\left(C_{j}\right) \rightarrow \tilde{\mu}_{\infty}\left(C_{j}\right)$ and $\tilde{\mu}_{n}\left(\left\{a_{k}\right\}\right) \rightarrow \tilde{\mu}_{\infty}\left(\left\{a_{k}\right\}\right)$ for all $j \geq 1$ and all $k \geq 0$ with

$$
\sum_{j=0}^{\infty} \tilde{\mu}_{n}\left(C_{j}\right)+\sum_{k=0}^{\infty} \tilde{\mu}_{n}\left(\left\{a_{k}\right\}\right)=\sum_{j=0}^{\infty} \tilde{\mu}_{\infty}\left(C_{j}\right)+\sum_{k=0}^{\infty} \tilde{\mu}_{\infty}\left(\left\{a_{k}\right\}\right)=1
$$

then by Scheffe's Theorem (Billingsley, 1968, p224), $\sum_{j=1}^{\infty}\left|\tilde{\mu}_{n}\left(C_{j}\right)-\tilde{\mu}_{\infty}\left(C_{j}\right)\right|$ and $\sum_{k=0}^{\infty}\left|\tilde{\mu}_{n}\left(\left\{a_{k}\right\}\right)-\tilde{\mu}_{\infty}\left(\left\{a_{k}\right\}\right)\right|$ converge both to zero. We have then, for $n$ sufficiently large

$$
\left|\int f h_{n} d \lambda_{n}-\int f h_{n} d \lambda_{\infty}\right| \leq M B \varepsilon
$$


Finally, we have

$$
\begin{aligned}
& \left|\int f h_{n}\left(W_{T}\right) d \lambda_{\infty}-\int f h_{\infty}\left(W_{T}\right) d \lambda_{\infty}\right| \\
& \leq \sum_{j=0}^{N^{\varepsilon}}\left|\int_{\tilde{C}_{j}} f h_{n}\left(W_{T}\right) d \lambda_{\infty}-\int_{\tilde{C}_{j}} f h_{\infty}\left(W_{T}\right) d \lambda_{\infty}\right| \\
& +\sum_{j=N^{\varepsilon}+1}^{\infty}\left|\int_{\tilde{C}_{j}} f h_{n}\left(W_{T}\right) d \lambda_{\infty}-\int_{\tilde{C}_{j}} f h_{\infty}\left(W_{T}\right) d \lambda_{\infty}\right| \\
& +M\left(\sum_{k=0}^{N^{\varepsilon}}\left|h_{n}\left(a_{k}\right)-h_{\infty}\left(a_{k}\right)\right|+\sum_{k=N^{\varepsilon}+1}^{\infty}\left|h_{n}\left(a_{k}\right)-h_{\infty}\left(a_{k}\right)\right|\right) \lambda_{\infty}\left(\left\{y_{k}\right\}\right) \\
& \leq M \sum_{j=0}^{N^{\varepsilon}}\left|h_{n}\left(u_{j}\right)-h_{\infty}\left(u_{j}\right)\right| \tilde{\mu}_{\infty}\left(C_{j}\right)+2 M B \sum_{j=N^{\varepsilon}+1}^{\infty} \tilde{\mu}_{\infty}\left(C_{j}\right) \\
& +2 M B \sum_{k=N^{\varepsilon}+1}^{\infty} \tilde{\mu}_{\infty}\left(\left\{a_{k}\right\}\right)+M \sum_{k=0}^{N^{\varepsilon}}\left|h_{n}\left(a_{k}\right)-h_{\infty}\left(a_{k}\right)\right| \tilde{\mu}_{\infty}\left(\left\{a_{k}\right\}\right) .
\end{aligned}
$$

The sum of the second and the third term is lower than $2 M B \varepsilon$, furthermore, for all $j$ and all $k$,

$$
\begin{aligned}
\left|h_{n}\left(u_{j}\right)-h_{\infty}\left(u_{j}\right)\right| \tilde{\mu}_{\infty}\left(C_{j}\right) & \leq 2 B \tilde{\mu}_{\infty}\left(C_{j}\right), \quad\left|h_{n}\left(u_{j}\right)-h_{\infty}\left(u_{j}\right)\right| \tilde{\mu}_{\infty}\left(C_{j}\right) \rightarrow 0 \\
\left|h_{n}\left(a_{k}\right)-h_{\infty}\left(a_{k}\right)\right| \tilde{\mu}_{\infty}\left(\left\{a_{k}\right\}\right) & \leq 2 B \tilde{\mu}_{\infty}\left(\left\{a_{k}\right\}\right), \quad\left|h_{n}\left(a_{k}\right)-h_{\infty}\left(a_{k}\right)\right| \tilde{\mu}_{\infty}\left(\left\{a_{k}\right\}\right) \rightarrow 0 \\
1 & =\sum_{j} \tilde{\mu}_{\infty}\left(C_{j}\right)+\sum_{k} \tilde{\mu}_{\infty}\left(\left\{a_{k}\right\}\right)
\end{aligned}
$$

We have then, by the Weierstrass M-test (see Billingsley (1995, Theorem A28, p. 543) that

$$
\sum_{j=1}^{N^{\varepsilon}}\left|h_{n}\left(u_{j}\right)-h_{\infty}\left(u_{j}\right)\right| \tilde{\mu}_{\infty}\left(C_{j}\right)+\sum_{k=1}^{N^{\varepsilon}}\left|h_{n}\left(a_{k}\right)-h_{\infty}\left(a_{k}\right)\right| \tilde{\mu}_{\infty}\left(\left\{a_{k}\right\}\right) \rightarrow 0
$$

and, for $n$ sufficiently large, we have then

\section{- Step 4 : Back to the original measures}

$$
\left|\int f h_{n}\left(W_{T}\right) d \lambda_{\infty}-\int f h_{\infty}\left(W_{T}\right) d \lambda_{\infty}\right|<2 M \varepsilon(1+B) .
$$

In conclusion, we have

$$
\begin{aligned}
& \left|\int f h_{n}\left(W_{T}\right) d \mu_{n}-\int f h_{\infty}\left(W_{T}\right) d \mu_{\infty}\right| \\
& \leq\left|\int f h_{n}\left(W_{T}\right) d \mu_{n}-\int f h_{n}\left(W_{T}\right) d \lambda_{n}\right|+\left|\int f h_{n}\left(W_{T}\right) d \lambda_{\infty}-\int f h_{n}\left(W_{T}\right) d \lambda_{n}\right| \\
& +\left|\int f h_{n}\left(W_{T}\right) d \lambda_{\infty}-\int f h_{\infty}\left(W_{T}\right) d \lambda_{\infty}\right|+\left|\int f h_{\infty}\left(W_{T}\right) d \mu_{\infty}-\int f h_{\infty}\left(W_{T}\right) d \lambda_{\infty}\right| \\
& <\varepsilon M(4+7 B)
\end{aligned}
$$


We have then proved that

$$
\int f_{n} h_{n}\left(W_{T}\right) d \mu_{n} \rightarrow \int f_{\infty} h_{\infty}\left(W_{T}\right) d \mu_{\infty} .
$$

\section{5 $Q_{\infty}$ is a martingale-measure for $X_{\infty}$}

Applying last Lemma to our family of probability measures $\left(Q_{n}\right)$ or more precisely to their densities $\left(g_{n}\right)$ with respect to the probability measures $\mu_{n}$ and to a constant sequence $(f)$, we obtain that for or a given subsequence that we will continue to denote by $\left(g_{n}\right)$ and for any bounded random variable $f$ on $\mathbb{D}^{m}$,

$$
\int f g_{n}\left(W_{T}\right) d \mu_{n} \rightarrow \int f g_{\infty}\left(W_{T}\right) d \mu_{\infty}
$$

Or, in other words,

$$
\int f(u) d \tilde{Q}_{n} \rightarrow \int f(u) g_{\infty}(u) d \mu_{\infty}
$$

It is easy then to conclude that $g_{\infty}$ is the density of $\tilde{Q}_{\infty}$ with respect to $\mu_{\infty}$. Furthermore, we know that, for $n \in \mathbb{N}, X_{n}$ is a martingale with respect to $Q_{n}$ and for $n=1, \cdots, \infty$, it admits $\tilde{Q}_{n}$ as a distribution probability with respect to $Q_{n}$. The sequence $\left(X_{n}, Q_{n}\right)$ converges then in distribution to $\left(X_{\infty}, Q_{\infty}\right)$ where $Q_{\infty}$ is the probability measure with density $g_{\infty}\left(X_{\infty}(T)\right)$ with respect to $P_{\infty}$.

The probability-measure $Q_{\infty}$ is then a martingale-measure for $X_{\infty}$ with respect to the filtration generated by $X_{\infty}$ (see Jacod and Shiryaev, Proposition 1.1, Chapter IX, p481).

\subsection{Back to the original sequence of processes}

If we reintroduce our notations with $b$, we proved that the stopped process $X_{\infty}^{b}$ is a martingale with respect to the filtration that it generates and the probability $Q_{\infty}^{b}$ with density $g_{\infty}^{b}\left(W\left(X_{\infty}^{b}(T)\right)\right)$ with respect to $P_{\infty}$.

Therefore $X_{\infty}^{b}$ is a local martingale for all the probability-measures with density $g_{\infty}^{b^{\prime}}\left(W\left(X_{\infty}^{b^{\prime}}(T)\right)\right)$ for all $b^{\prime}>b$. Furthermore $X_{\infty}^{b}$ is bounded and $X_{\infty}$ is locally bounded. Therefore, for $b^{\prime}$ sufficiently large $P_{\infty}\left(g_{\infty}^{b^{\prime}}\left(W\left(X_{\infty}^{b^{\prime}}(T)\right)\right)=g_{\infty}\left(W\left(X_{\infty}(T)\right)\right)\right)$ is arbitrarily close to 1 . The process $X_{\infty}^{b}$ is then a local-martingale for the probabilitymeasure with density $\left.g_{\infty}\left(W\left(X_{\infty}(T)\right)\right)\right)$ and so is the process $X_{\infty}$.

If we apply again the result of our Lemma to the sequence $\left(F_{n}\right)$ which defines our derivative assets, we have

$$
\int F_{n} g_{n}\left(W_{T}\right) d \mu_{n} \rightarrow \int F_{\infty} g_{\infty}\left(W_{T}\right) d \mu_{\infty}
$$

or equivalently

$$
\int F_{n}\left(X_{n}\right) d Q_{n} \rightarrow \int F_{\infty}\left(X_{\infty}\right) d Q_{\infty}
$$

where $\int F\left(X_{n}\right) d Q_{n}$ is in $E I_{n}\left(F_{n}\right)$ and $\int F\left(X_{\infty}\right) d Q_{\infty}$ is in $E I_{\infty}^{l o c, a c}\left(F_{\infty}\right)$. We proved then that

$$
E I_{n}\left(F_{n}\right) \hookrightarrow E I_{\infty}^{l o c, a c}\left(F_{\infty}\right) .
$$


If the set $E I_{\infty}(F)$ is nonempty, i.e. if it exists at least one absolutely-continuous, martingale measure with a density defined by a non-increasing function of the total wealth in the model $M_{\infty}$, it is easy then to see that we have

$$
E I_{n}(F) \hookrightarrow \overline{E I_{\infty}^{l o c}(F)} .
$$

If, finally $X_{\infty}$ is of class(D) with respect to $P_{\infty}$ and if all the assets are in positive supply, with respect to $P_{\infty}$ then, by definition, for all $\varepsilon$, there exists $k$, such that for all finite valued stopping time $\tau$,

$$
\int_{\left\|X_{\infty}(\tau)\right\| \geq b} X_{\infty}(\tau) d P_{\infty} \leq \varepsilon
$$

Since $g_{\infty}$ is bounded, we have then

$$
\int_{\left\|X_{\infty}(\tau)\right\| \geq b} X_{\infty}(\tau) g_{\infty}\left(W^{\tau}\right) d P_{\infty} \leq \varepsilon B
$$

or equivalently

$$
\int_{\left\|X_{\infty}(\tau)\right\| \geq b} X_{\infty}(\tau) d Q_{\infty}=\int_{\left\|X_{\infty}(\tau)\right\| \geq b} X_{\infty}(\tau) g_{\infty}\left(W^{\tau}\right) d P_{\infty} \leq \varepsilon B .
$$

The process $X_{\infty}$ is then of class (D) with respect to $Q_{\infty}$ and consequently a martingale with respect to $Q_{\infty}$ (Jacod and Shiryaev, 1987, Proposition 1-47, p11). We have then

$$
E I_{n}(F) \hookrightarrow \overline{E I_{\infty}(F)} .
$$

Assume now that we only have

$$
E I_{n}\left(F_{n}\right) \hookrightarrow E I_{\infty}^{l o c, a c}\left(F_{\infty}\right) .
$$

but that there exists only one equivalent martingale measure, then there exists only one equivalent local-martingale measure and consequently there exists only one absolutelycontinuous martingale measure (Jacod, 1979, Theorem 11-4). We have then $E I_{\infty}^{\text {loc,ac }}\left(F_{\infty}\right)=$ $E I_{\infty}\left(F_{\infty}\right)$ and is reduced to a single element, therefore $\lim _{n} E I_{n}\left(F_{n}\right)=E I_{\infty}\left(F_{\infty}\right)$.

Remark 2 If we want the equality in (i), we don't really need market completeness nor the uniqueness of the equivalent martingale measure. It suffices to impose that there is only one element in $E I_{\infty}^{l o c, a c}\left(F_{\infty}\right)$. This is, in particular, the case when there is only one absolutely-continuous local-martingale measure in "reverse order" than the total wealth. Such a situation may occur even in incomplete markets as in the following example.

Example 7 Consider a one step trinomial model with equiprobable returns $1+\delta \varepsilon$ with $\varepsilon>0$ and $\delta=-1,0,1$. This model is obviously incomplete. If we assume that there is a zero-interest rate, it is easy to check that there exists one and only one martingale-measure in reverse order of the stock prices, namely $(1 / 3,1 / 3,1 / 3)$. 


\section{References}

Aït-Sahalia, Y. and A. W. Lo (1997), "Nonparametric risk management and implied risk aversion", NBER WP 6130,.

Aït-Sahalia, Y. and A. W. Lo (1998), "Nonparametric estimation of state-price densities implicit in financial asset prices", Journal of Finance, 53, 499-547.1.

Avellaneda, M., A. Levy, and A. Paras (1995), "Pricing and hedging derivative securities in markets with uncertain volatilities", Applied Mathematical Finance, 2, 73-88.

Billingsley, P., (1968), Convergence of probability measures, John Wiley and Sons, New-York,

Billingsley, P. (1995), Probability and Measure, Third Edition, John Wiley and Sons, New-York,

Bizid, A., E. Jouini and P.F. Koehl (1998), "Pricing of nonredundant assets derivatives in a complete market", Review of Derivatives Research, 2, 285-312.

Black, F. and M. Scholes (1973), "The pricing of options and corporate liabilities", Journal of Political Economy 81 637-654.

Cox, J., S. Ross and M. Rubinstein, (1979), "Option Pricing: A Simplified Approach," Journal of Financial Economics 7, pp. 229-263.

Duffie, D. (1988), Security markets : stochastic models, Academic Press, Boston,

Duffie, D. and P. Protter (1992), "From discrete to continuous time finance : weak convergence of the financial gain process", Mathematical Finance, 2(1), pp1-15

Helly, E. (1912), "über lineare Funktionaloperationen", Sitzungsberichte der Kaiserlichen Akademie der Wissenschaften, Mathematisch-Naturwissenschaftliche Klasse, 121, 265-297.

Hubalek, F. and W. Schachermayer (1998), "When does convergence of asset price processes imply convergence of option prices", Mathematical Finance, 8(4), 385-403.

Jacod, J., (1979), Calcul stochastique et problèmes de martingales, Lectures Notes in Mathematics, Series : Institut de Mathé matiques, Université de Strasbourg, 714, Springer-Verlag.

Jacod, J. and A. Shiryaev, (1987), Limit theorems for stochastic processes, SpringerVerlag, Berlin, 
Jakubowski, Mémin and Pagès (1989), "Convergence en loi des suites d'intégrales stochastiques sur l'espace $G D^{1}$ de Skorokhod", Probab. Th. Rel. Fields, 81 (1989), 111-137.

Jouini and Napp (1999), "Continuous time pricing of nonredundant assets", Working Paper 9830, CREST,

Kurtz, T. and P. Protter (1991a), "Weak limit theorems for stochastic integrals and stochastic differential equations", Ann. Prob, 19, 1035-1070,

Kurtz and Protter (1991b), "Weak Convergence of Stochastic Integrals and Differential Equations", in Lecture Notes in Mathematics: Probabilistic Models for Nonlinear Partial Differential Equations (Springer).

Perrakis, S. (1986), "Option Bounds in Discrete Time: Extensions and the Pricing of the American Put," Journal of Business 59, pp. 119-141.

Perrakis, S. (1993), "Option pricing for multinomial stock returns in diffusion and mixed processes", Canadian Journal of Administrative Sciences, 10(1), 68-82.

Perrakis, S. and P.J. Ryan (1984), "Option Pricing Bounds in Discrete Time," Journal of Finance 39, pp. 519-525.

Prigent, J.-L. (1994), "Pricing of contingent claims from discrete to continuous time models : on the robustness of the Black and Scholes formula", Working Paper, THEMA, Université de Cergy-Pontoise, 11/94.1.

Ranga Rao, R., (1962), "Relations between weak and uniform convergence of measures with applications", Ann. Math. Statist. 33, 659-680. 